Document downloaded from:

http://hdl.handle.net/10251/160603

This paper must be cited as:

Larrainzar-Garijo, R.; Caeiro, J.; Marco, M.; Giner Maravilla, E.; Miguélez, M. (2019).

Validación experimental de un modelo de análisis de elementos finitos en fractura de cadera y su aplicabilidad clínica. Revista Española de Cirugía Ortopédica y Traumatología. 63(2):146-154. https://doi.org/10.1016/j.recot.2018.05.006

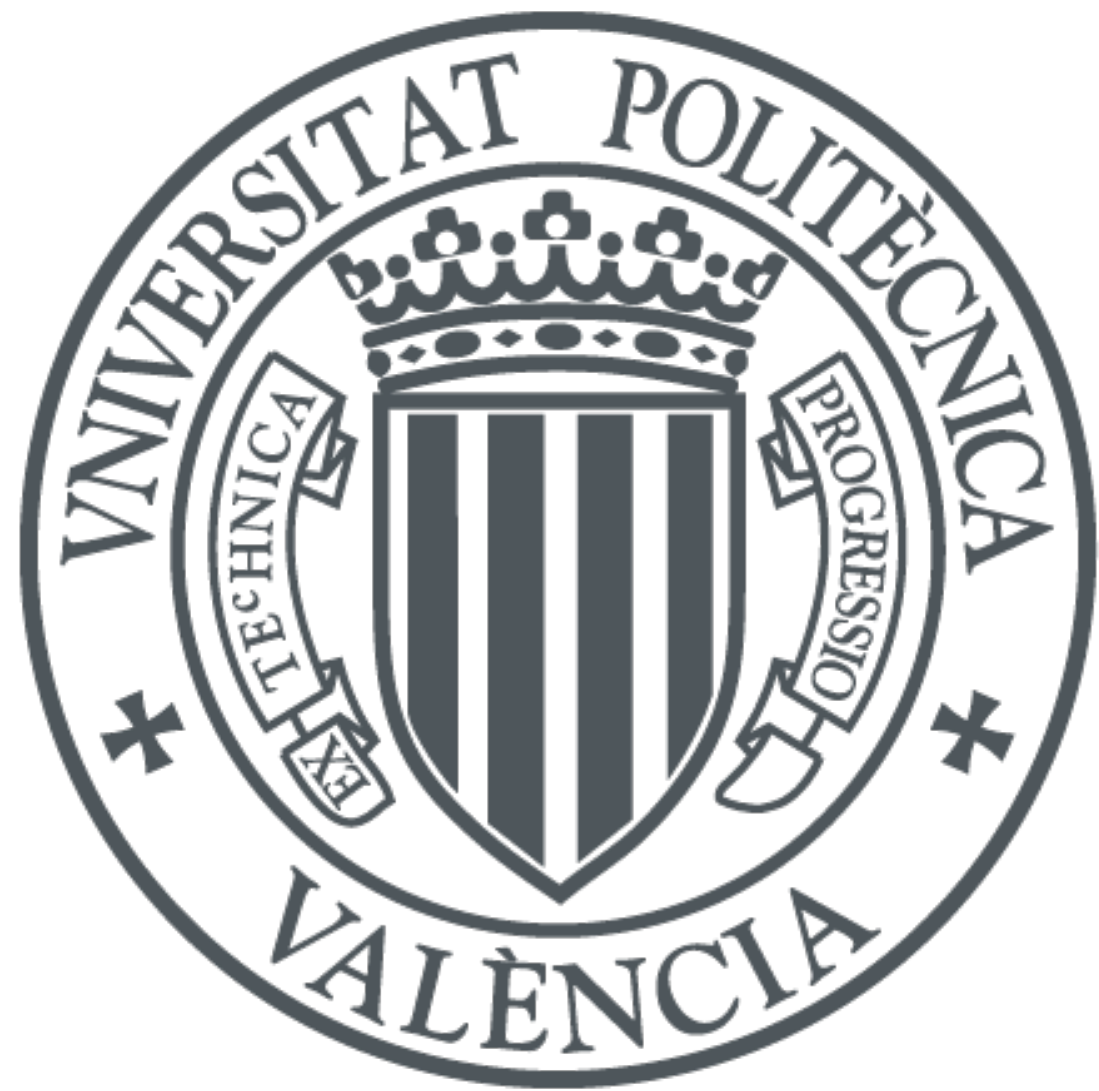

The final publication is available at

https://doi.org/10.1016/j.recot.2018.05.006

Copyright Elsevier

Additional Information 


\section{VALIDACIÓN EXPERIMENTAL DE UN MODELO DE ANALISIS DE ELEMENTOS FINITOS EN FRACTURA DE CADERA Y SU APLICABILIDAD CLÍNICA}

EXPERIMENTAL VALIDATION OF OF FINITE ELEMENTS MODEL IN HIP FRACTURE AND ITS CLINICAL APPLICABILITY

Ricardo Larrainzar-Garijo ${ }^{a, b}$, José Ramón Caeiro ${ }^{c}$, Miguel Marco Esteban ${ }^{d}$, Eugenio Giner ${ }^{e}$, María Henar Miguélez ${ }^{d}$

a Servicio de Cirugía Ortopédica y Traumatología. Hospital Universitario Infanta Leonor

b Departamento de Cirugía. Facultad Medicina, Universidad Complutense Madrid, España.

${ }^{c}$ Servicio de Cirugía Ortopédica y Traumatología Complejo Hospitalario Universitario de Santiago de Compostela

${ }^{d}$ Departamento de Ingeniería Mecánica Universidad Carlos III de Madrid $e$

Departamento de Ingeniería Mecánica y Materiales Universitat Politècnica de València - CIIM.

Los autores declaran no tener ningún conflicto de intereses en relación con

$$
\text { el manuscrito y sus conclusiones }
$$

Los autores quieren agradecer a la Dra Teresa Vázquez Profesora Titular del departamento de Anatomía de la Universidad Complutense de Madrid su ayuda para la consecución del trabajo

Correspondencia autor 
Profesor Asociado Cirugía. Facultad Medicina Universidad Complutense Madrid Hospital Universitario Infanta Leonor

C/ Gran Vía del Este, 80

28031 Madrid

Tel: 911918946

FAX: 911919075

E-mail: rlarrainzar@gmail.com 


\section{VALIDACIÓN EXPERIMENTAL DE UN MODELO DE ANALISIS DE ELEMENTOS FINITOS EN FRACTURA DE CADERA Y SU APLICABILIDAD CLÍNICA}

La fractura de la extremidad proximal de fémur es objeto de interés en investigación. La complejidad del entramado óseo y la ineficiencia estructural asociada al envejecimiento hacen que existan muchas variables todavía por comprender desde el punto de vista experimental pero no existe un modelo de investigación estructural y biomecánico de la fractura de cadera claramente definido

\section{MATERIAL Y METODO}

Trabajo experimental sobre cadáver y numérico basado en elementos finitos.Las relaciones establecidas entre las unidades Hounsfield, densidad mineral ósea (DMO) y propiedades mecánicas (módulo de Young) de cada voxel, la simulación de carga y las sujeciones distales del fémur proximal virtual se aplicaron de forma similar a lo realizado en los ensayos experimentales previos. La propagación de la fractura se modeló mediante desarrollo computacional propio del equipo investigador, disminuyendo las propiedades mecánicas de los elementos dañados, simulando así la separación entre las distintas partes fracturadas.

\section{RESULTADOS}

El modelo computacional fue capaz de determinar el punto de inicio de la fractura, con una discreta tendencia a la medialización anatómica de dicho punto.El grado de correlación fue muy alto al comparar el valor real de deformación de las muestras frente al obtenido por el modelo computacional.Sobre 32 puntos analizados se obtuvo una pendiente de 1.03 en regresión lineal,con un error relativo entre las deformaciones del $6 \%$ y un coeficiente de Pearson de $R^{2}=0.99$.

\section{CONCLUSION}

El principal logro de este trabajo es haber desarrollado un modelo de AEF del fémur proximal capaz de simular e imitar el comportamiento biomecánico de fémures humanos contrastado frente a un modelo experimental clásico realizado en piezas anatómicas. Sobre esta base podrán evaluarse interacciones cualitativas y cuantitativas que lo consoliden como un potente banco de ensayos de experimentación computacional sobre el fémur proximal humano. 


\section{INTRODUCCIÓN}

La fractura de la extremidad proximal de fémur presenta interés desde cualquier punto de vista ya sea clínico o socioeconómico. La alta mortalidad asociada, la dependencia que genera y el alto coste económico del proceso asistencial condicionan que el foco de la comunidad científica se haya centrado, casi exclusivamente, en el estudio del enfermo afecto de fractura. Sin embargo se desconocen aspectos intrínsecos (patogénicos y biomecánicos) de la fractura de la extremidad proximal del fémur: el punto de inicio de la fractura, la propagación de la misma, qué variables condicionan los distintos patrones de fractura, etc. La complejidad del entramado óseo y la ineficiencia estructural asociada al envejecimiento, que convierten al hueso en un material más frágil para soportar cargas e impactos, hacen que existan muchas variables todavía por comprender desde el punto de vista experimental pero no existe un modelo de investigación estructural y biomecánico de la fractura de cadera claramente definido.(1-4)

Tampoco existe consenso sobre si el estudio a través de estas piezas anatómicas reales debe realizarse sobre hueso fresco o hueso seco y que cambios estructurales puedan llevar implícitos(5) y ello ha llevado a la alternativa de realizar el trabajo experimental de campo a través de huesos sintéticos(5-9) que, simulando las características microestructurales de cortical y esponjosa, permitan abaratar las inversiones necesarias. Este material de trabajo proporcionado por fabricantes simulando el hueso humano ha permitido avanzar en el conocimiento mecánico del hueso sano, pero difícilmente es extrapolable al mundo clínico. La producción industrial a escala de los especímenes sintéticos solo permite elaborar fantomas estándar de hueso sano o fantomas estándar de hueso osteoporótico

Por ello, los modelos numéricos basados en AEF se plantean como una alternativa válida ya que gracias al aumento del poder computacional disponible en la actualidad el investigador puede someter a cargas a estructuras "virtuales" sin la necesidad de disponer de un material físico, con la ventaja que aporta el poder cambiar los parámetros estructurales que más afectan al hueso como estructura de soporte de 
cargas: densidad mineral ósea (DMO), área de sección, grosor cortical, porosidad cortical, microestructura trabecular, etc $(1,8,10-12)$

El análisis mediante elementos finitos (AEF) se basa en la descomposición de una estructura compleja, de comportamiento biomecánico desconocido, en una serie de subestructuras geométricamente simplificadas denominadas elementos finitos. El comportamiento individual de cada una de estas subestructuras, denominadas "ladrillos", se determina mediante ecuaciones matemáticas que evalúan las cargas o las tensiones que estas reciben. A partir del análisis individual de cada uno de estos elementos, se obtiene un modelo computacional global de la estructura analizada, que permite por un lado determinar su resistencia global y, por otro, ser "sometido a carga" matemáticamente para determinar la forma, el tipo y la localización del fracaso biomecánico

Se han desarrollado modelos vertebrales $(13)$ y femorales $(1,6,14)$ de AEF a partir de cortes 3D de tomografía cuantitativa de alta resolución (AR-QCT) y también de imágenes de resonancia magnética nuclear (RMN) de alta resolución. Los modelos obtenidos mediante estos análisis son muy exactos incluso cuando se emplean resoluciones de escaneado bajas.(15) Se ha demostrado que existe una buena correlación entre el comportamiento biomecánico y la resistencia ósea total predicha por $\operatorname{AEF}(16,17)$ y los determinados experimentalmente en especímenes vertebrales y femorales de cadáver de manera que este tipo de análisis demuestra incluso mayor capacidad que la densidad mineral ósea volumétrica (VDMO) y el área de la sección transversal (AST) obtenibles por HR-QCT para predecir la resistencia a la compresión $(1,15,18,19)$. Si bien estas correlaciones resultan muy interesantes para la evaluación in vitro de la resistencia total de un hueso, el cálculo mediante AEF de la resistencia ósea in vivo tal vez no logre conseguir unas correlaciones tan altas, posiblemente debido a las complejas interacciones entre hueso, músculo y vectores de carga(20).

Pero además existe una dificultad añadida para la transferencia clínica de estos resultados ya que los entornos de computación son controlados por el propio equipo investigador, precisando validación de las predicciones computacionales. Muchas de las variables y parámetros se toman de referencias de trabajos experimentales 
previamente validados y publicadas, relacionados generalmente con las características de un hueso envejecido y/o con osteoporosis senil. (16)

Otra limitación importante de los modelos AEF publicados hasta la fecha es que consideran al hueso como un material homogéneo en lo que se refiere cuanto a las propiedades biomecánicas relacionadas con la fractura, y no hay nada más alejado de la realidad(6,21). Cualquier modelo matemático que no contemple este hecho nunca podrá tener aplicabilidad clínica, fin último de la investigación biomédica.

Por todo ello es necesario determinar nuevos modelos de investigación basados en $A E F$ que reúnan el conocimiento experimental de los últimos estudios mecánicos con la potencia que brinda las actuales capacidades de parametrización computacional.

La hipótesis de este trabajo es que es posible desarrollar un modelo de experimentación computacional que caracterice el hueso de la extremidad proximal del fémur como un material heterogéneo a partir de la traslación directa de los parámetros mecánicos obtenidos de piezas anatómicas de experimentación.

\section{MATERIAL Y MÉTODO}

\section{Especímenes}

El trabajo experimental y numérico ha sido llevado a cabo sobre dos fémures humanos, donados por el departamento de Anatomía de la Universidad Complutense de Madrid, pertenecientes al Centro de Donación de cuerpos y salas de disección de dicha Universidad. La tabla 1 muestra los datos antropométricos de los donantes. Los sujetos no tenían patologías músculo esqueléticas conocidas.

Tabla 1: Características generales de los especímenes estudiados

Se efectuó disección anatómica del miembro inferior de los cadáveres con desinserción total de todas las estructuras cápsulo-ligamentosas coxofemorales y de rodilla hasta la extracción del fémur total sin inserciones osteotendinosas. Se realizó osteotomía transversa de la extremidad proximal del fémur a una distancia de $35 \mathrm{~cm}$ 
del punto más distal del trocánter menor. Las piezas se conservaron frescas en material humedecido en suero salino hasta la realización de la tomografía computerizada (TC) 12 horas más tarde.

\section{Obtención del modelo geométrico 3D}

Se realizó una AR-QCT a cada uno de los especímenes femorales mediante un escáner clínico convencional modelo Siemens Somaton, Los fémures se dispusieron en posición horizontal fijados por su parte más distal, con una orientación del cuello femoral paralela al suelo y una superficie libre de material óseo de $25 \mathrm{~cm}$ en el arco de escaneado. La resolución del estudio fue de $0.44 \mathrm{~mm}$ en el plano transversal y $1.0 \mathrm{~mm}$ en la dirección longitudinal del fémur, al objeto de obtener voxeles con dimensiones de $0.2 \times 0.2 \times 0.2 \mathrm{~mm}^{3}$. El tratamiento de la imagen DICOM obtenida y la su posterior segmentación se llevó a cabo mediante el software ScanIP (Simpleware, Exeter, UK). Esta metodología permitió la generación del volumen geométrico correspondiente al fémur proximal, la discriminación de sus dos principales materiales (hueso cortical y hueso trabecular), densidades radiológicas y la caracterización mecánica de cada espécimen.

\section{Modelo numérico de elementos finitos del fémur proximal}

Un análisis previo de la sensibilidad del mallado permitió establecer una malla de 3 $\mathrm{mm}$ en la zona de la diáfisis y $2 \mathrm{~mm}$ en la zona proximal, con un refinamiento en el cuello femoral de $1 \mathrm{~mm}$.

Con estas condiciones, y mediante el código de elementos finitos (EF) Abaqus/Standard, se generó la malla del fémur proximal formada por 500000 elementos tetraédricos cuadráticos y unos 1000000 nodos. La figura 1 muestra la malla obtenida.

Figura 1: Modelo 3D virtual por AEF de los especímenes estudiados

Las relaciones establecidas entre las unidades Hounsfield, densidad mineral ósea (DMO) y propiedades mecánicas (módulo de Young) de cada voxel se establecieron mediante las ecuaciones publicadas por Morgan et al(22). La simulación de carga y 
las sujeciones distales del fémur proximal virtual se aplicaron de forma similar a lo realizado en los ensayos experimentales previos.

La propagación de la fractura se modeló mediante desarrollo computacional propio del equipo investigador, disminuyendo las propiedades mecánicas de los elementos dañados, simulando así la separación entre las distintas partes fracturadas. Para el cálculo de la carga máxima de fractura se han considerado los valores críticos de tensión en cada elemento en función de su DMO.

\section{Modelo experimental de fémur proximal}

Para la adaptación de las piezas anatómicas al banco de ensayos se diseñó un utillaje especial formado por dos conjuntos de piezas fabricados en una aleación de aluminio (AA 7075-T6). El conjunto superior estaba formado por una placa para sujetar el útil a la prensa y un cilindro con un extremo cóncavo para adaptarse a la geometría de la cabeza del fémur con un radio de $25 \mathrm{~mm}$. El conjunto inferior estaba formado por una caja en la que se embebe la diáfisis del fémur mediante polimetilmetacrilato (PMMA) y una placa para fijarla a la máquina de ensayos. La caja tiene una cuña para que la geometría resultante forme 8 o con el eje del fémur. La figura 2 muestra los diseños específicos desarrollados

Figura 2: Utillaje de fabricación propia para la realización del trabajo de campo experimental.

Se realizaron dos ensayos experimentales consecutivos sobre cada pieza anatómica. El primero consistió en aplicar fuerzas progresivas de carga en el plano frontal iguales a $500 \mathrm{~N}, 1000 \mathrm{~N}, 1500 \mathrm{~N}$ y $2000 \mathrm{~N}$, con el eje de carga a 8 o con respecto al de la diáfisis femoral. Posteriormente se realizó un aumento progresivo de la carga sobre la cabeza del fémur hasta que se produjo la fractura macroscópica de la extremidad proximal.

Para cuantificar las deformaciones experimentadas por la pieza durante las cargas mencionadas se dispusieron galgas extensiométricas sobre la superficie del fémur, dos 
en la diáfisis y una en el cuello femoral. La figura 3 muestra los especímenes en el banco de ensayos.

Figura 3: Espécimen sobre banco de pruebas experimental con las galgas colocadas para registro de las fuerzas de rotura.

\section{Variables de estudio}

Para la validación de la capacidad de predicción del modelo numérico se consideraron 4 variables a comparar entre el modelo experimental y computacional:

- Punto de inicio de la fractura

- Propagación de la fractura y correlación con patrón clínico

- Deformaciones obtenidas en cargas inferiores a la fractura

- Carga máxima de fractura

\section{RESULTADOS}

\section{Punto de inicio de la fractura}

El modelo computacional fue capaz de determinar el punto de inicio de la fractura, con una discreta tendencia a la medialización anatómica de dicho punto (Tabla 2)

Tabla 2 Distancia en milímetros entre el punto de inicio de fractura y el ápex del trocanter mayor.

\section{Propagación de la fractura}

La línea de propagación del modelo computacional en los dos especímenes tuvo un patrón cuasi superponible a los obtenidos en los modelos experimentales como muestra la figura 4 
Figura 4: Se observa la propagación de la propagación de la fractura comparando el experimental real con la computacional ( $A$ y $C$; $B$ y D). En $C$ se observa una doble progresión de la fractura pero con un único punto de inicio subcapital

\section{Deformaciones obtenidas en cargas inferiores a la fractura}

El grado de correlación fue muy alto al comparar el valor real de deformación de las muestras frente al obtenido por el modelo computacional,.

Para un total de 32 puntos analizados ( 4 deformaciones por 4 casos de carga en cada fémur), se obtuvo una pendiente de 1.03 en la regresión lineal, con un error relativo entre las deformaciones del $6 \%$ y un coeficiente de Pearson de $\mathrm{R}^{2}=0.99$. Figura 5

Figura 5: muestra la comparación entre las deformaciones en ambos sujetos.

\section{Carga máxima de fractura}

Tanto en el modelo computacional como en el experimental, el valor de carga máxima era mayor en el espécimen 2, correspondiente a un varón, con una diferencia aproximada de $1000 \mathrm{~N}$ entre los dos donantes.

El modelo computacional de EF infraestimó discretamente la carga máxima de fractura, con un error relativo aproximado al $10 \%$. La tabla 3 muestra los valores numéricos de carga de fractura.

Tabla 3 Valor en Newton de la carga para conseguir una fractura.

\section{DISCUSIÓN}

El principal logro de este trabajo es haber desarrollado un modelo de computacional de AEF del fémur proximal capaz de simular e imitar el comportamiento biomecánico de fémures humanos, tanto para cargas bajas, como para cargas de fractura, que ha podido ser contrastado frente a un modelo experimental clásico realizado en piezas anatómicas sobre un banco de ensayos. Sobre este modelo computacional podrán 
evaluarse infinidad de interacciones cualitativas y cuantitativas por el equipo investigador: condiciones biomecánicas de precarga, condiciones de carga, modelos de fracturas, material de osteosíntesis, implantes protésicos, etc., que lo consoliden como un potente banco de ensayos de experimentación computacional sobre el fémur proximal humano.

Para que el modelo computacional de un fémur proximal sea lo más realista posible, y especialmente en el momento de evaluar su comportamiento en un modelo de fractura por fragilidad, es necesario que sus propiedades mecánicas estén relacionadas básicamente con la $\operatorname{DMO}(1,20)$. En nuestro modelo, mediante filtros de software específicos, se ha podido diferenciar las distintas zonas del fémur dependiendo del valor de las unidades Hounsfield de cada voxel y, secundariamente por tanto, de la DMO. De esta manera se pudo, por un lado, diferenciar hueso trabecular de hueso cortical $y$, por otro, se pudo aplicar al modelo distintas propiedades mecánicas dependiendo de si el hueso presentaba o no una mayor DMO. De forma complementaria, en nuestro modelo se emplearon las relaciones de Morgan et al(22). para obtener la rigidez en cada elemento en función de su DMO. Y para el cálculo de la carga máxima de fractura se consideraron los valores críticos de tensión en cada elemento en función también de su DMO. Esta estrategia aproxima el modelo a las condiciones mecánicas que se dan el hueso osteoporótico senil, ofreciendo unos valores numéricos de cálculo lo más realistas posibles.

Pero para que el modelo computacional de AEF tenga aplicabilidad clínica es necesario que contenga además otras variables que, en base al conocimiento actual, influyen en el hueso como estructura de carga:

- El modelo numérico ha de ser heterogéneo tanto en la rigidez de los materiales como en las propiedades de fractura(21), con diferentes relaciones entre estos parámetros y la $\mathrm{DMO}(23)$.

- Un modelo adecuado de propagación de la fractura, en nuestro caso desarrollado a partir de un software propio(6), que demuestre una buena correlación entre lo obtenido en los test experimentales y la predicción computacional. 
- Aunque algunos trabajos de investigación analizan exclusivamente la respuesta de puntos o regiones aisladas del fémur, lo cual suele dar cargas numéricas inferiores a la experimental, la carga máxima (y la energía total) de fractura ha de obtenerse a partir de la curva de carga-desplazamiento, tal y como ocurre en un ensayo experimental real(1,12).

En este sentido, nuestro modelo es el más completo publicado hasta el momento, al tener en cuenta todas estas circunstancias. Creemos que la correlación estadística tan importante que existe entre deformidades obtenidas en cargas inferiores a la fractura entre el modelo computacional y lo obtenido experimentalmente en el banco de ensayos junto con la similitud de los trazos propagación de fractura de los especímenes 1 y 2 constituye un claro elemento de solidez del modelo computacional y es un hecho validador de nuestro modelo de elementos finitos de fémur proximal.

Queremos llamar la atención sobre que las condiciones de trabajo experimental para los dos especímenes han sido exactamente las mismas y controladas en todo momento por el equipo investigador (fuerzas progresivas de la carga en el plano frontal formando 8o con el eje de la diáfisis de 500 N, 1000 N, 1500 N y 2000 N) y por ello resulta llamativo que se produzcan dos tipos de rotura totalmente diferenciables entre sí como muestra la figura 4 y con importante similitud a lo que ocurre en la práctica clínica habitual. En el espécimen 1 se produce un trazo de fractura que puede clasificarse como subcapital mientras que en el espécimen 2 se trata de una fractura claramente extracapsular. Más sorprendente es, si cabe, que en la predicción computacional encontremos los mismos hallazgos. Por ello es sugestivo pensar que existen unos condicionamientos materiales y/o nano, micro y macro estructurales que condicionan un patrón de fractura independientemente de la intensidad de la carga o la dirección de aplicación de la misma. Ni la metodología empleada ni el número de especímenes analizados nos permite establecer una relación directa entre estas variables, pero si creemos que estos hallazgos refuerzan la exactitud de la predicción del modelo computacional y por ello su futura aplicabilidad clínica.

La principal limitación del modelo computacional para su utilización como herramienta de apoyo clínico son las inherentes al trabajo por EF. Como hemos comentado existe todo un proceso que incluye la traslación de un elemento físico real a un elemento 
virtual segmentado en pequeñas formas geométricas y que toda la relación de estos elementos entre sí está controlada por las condiciones que establece el equipo investigador.

También es cierto que se ha realizado trabajo anatómico, computacional y experimental solo sobre dos especímenes, pero es innegable que el número de donantes y la cantidad de recursos físicos y humanos que se necesitan para ampliar la serie es ingente, y que en otros sectores productivos y de investigación, como la ingeniería, el trabajo exclusivamente computacional constituye el patrón estándar en aquellas circunstancias donde los modelos experimentales clásicos son inviables o económicamente insostenibles $(8,11,24)$.

El modelo que presentamos representa la consolidación de una línea de trabajo de un grupo multidisciplinar compuesto por cirujanos ortopédicos e ingenieros con una clara y convencida orientación clínica $(6,21,23,25)$. Solo a través del trabajo colaborativo entre las distintas disciplinas del conocimiento humano podremos desarrollar estrategias que permitan el intercambio traslacional de pensamientos para el mejor cuidado de nuestros enfermos.

Únicamente con un conocimiento claro de las circunstancias mecánicas que condicionan la aparición de una fractura de la extremidad proximal de fémur estaremos en condiciones de hacer investigación traslacional hacia los aspectos clínicos más relevantes de esta patología: punto de inicio de la fractura y su posterior progresión a lo largo de la superficie ósea tipo de fractura, factores biomecánicos predictores de fractura y de su tipo, diseños específicos de implantes, factores predictores de fracaso de osteosíntesis, etc. $(1,10,14), \ldots \ldots$.

En relación con este último punto, los trabajos de Lenich $(26,27)$ han demostrado que el primer efecto que ocurre en el fenómeno de "cut out" es un momento de rotación del fragmento óseo sobre el implante cefálico para posteriormente, a través de un fenómeno continuo de micromovimientos ir dañando el hueso hasta que se produce la protrusión del material de osteosíntesis. El modelo computacional que hemos desarrollado, puede ayudar a entender este modelo patogénico, al permitir simular las alteraciones que se producirían al considerar como punto fijo el ápex de la circunferencia de la cabeza femoral y simular los micromovimientos de flexoextensión. 
En resumen, este es un artículo escrito por clínicos para clínicos en el que no se pretende, ni se quiere, entrar en discusión profunda sobre los fundamentos matemáticos que sustentan el método de $A E F$, ni en las fórmulas matemáticas que definen cada una de las relaciones de los elementos entre sí; pero en el que queremos destacar que la metodología que hemos utilizado para la obtención de la estructura geométrica "virtual" del fémur proximal, las condiciones materiales y estructurales previas, de contorno y de carga de fractura son las reales, y que el AEF es un método válido avalado en la literatura que viene siendo utilizado por equipos investigadores para la evaluación de distintas patologías relacionadas con nuestra especialidad.(7,11,13,23,28-31)

El modelo computacional de AEF desarrollado por este equipo investigador multidisciplinar se puede considerar, en conjunto, un modelo completo de AEF de la extremidad proximal del fémur con aplicabilidad clínica futura.

\section{BIBLIOGRAFIA}

1. Cristofolini L, Juszczyk M, Martelli S, Taddei F, Viceconti M. In vitro replication of spontaneous fractures of the proximal human femur. J Biomech. 2007;40(13):2837-45.

2. Santoni BG, Nayak AN, Cooper SA, Smithson IR, Cox JL, Marberry ST, et al. Comparison of Femoral Head Rotation and Varus Collapse Between a Single Lag Screw and Integrated Dual Screw Intertrochanteric Hip Fracture Fixation Device Using a Cadaveric Hemi-Pelvis Biomechanical Model. J Orthop Trauma. 2016;30(4):164-9.

3. Haynes RC, Pöll RG, Miles AW, Weston RB. Failure of femoral head fixation: a cadaveric analysis of lag screw cut-out with the gamma locking nail and AO dynamic hip screw. Injury. 1997;28(5-6):337-41.

4. Krischak GD, Augat P, Beck A, Arand M, Baier B, Blakytny R, et al. Biomechanical comparison of two side plate fixation techniques in an unstable intertrochanteric osteotomy model: Sliding Hip Screw and Percutaneous 
Compression Plate. Clin Biomech. 2007;22(10):1112-8.

5. Basso T, Klaksvik J, Syversen U, Foss OA. A biomechanical comparison of composite femurs and cadaver femurs used in experiments on operated hip fractures. J Biomech. 2014;47(16):3898-902.

6. Marco M, Giner E, Larraínzar R, Caeiro JR, Miguélez MH. Numerical Modelling of Femur Fracture and Experimental Validation Using Bone Simulant. Ann Biomed Eng. 2017;1-14.

7. Loh BW, Stokes CM, Miller BG, Page RS. Femoroacetabular impingement osteoplasty. Bone Joint J. 2015;97-B(9):1214-9.

8. Tsai AG, Reich MS, Bensusan J, Ashworth T, Marcus RE, Akkus O. A fatigue loading model for investigation of iatrogenic subtrochanteric fractures of the femur. Clin Biomech. 2013;28(9-10):981-7.

9. Knobe M, Altgassen S, Maier K-J, Gradl-Dietsch G, Kaczmarek C, Nebelung S, et al. Screw-blade fixation systems in Pauwels three femoral neck fractures: a biomechanical evaluation. Int Orthop. 2018;42(2):409-18.

10. García-Aznar JM, Bayod J, Rosas A, Larrainzar R, García-Bógalo R, Doblaré M, et al. Load Transfer Mechanism for Different Metatarsal Geometries: A Finite Element Study. J Biomech Eng. 2008;131(2):2101-1-7.

11. Cilla $\mathrm{M}$, Checa S, Preininger B, Winkler T, Perka C, Duda GN, et al. Femoral head necrosis: A finite element analysis of common and novel surgical techniques. Clin Biomech. 2017;48:49-56.

12. Schileo E, Taddei F, Cristofolini L, Viceconti M. Subject-specific finite element models implementing a maximum principal strain criterion are able to estimate failure risk and fracture location on human femurs tested in vitro. J Biomech. 2008;41(2):356-67.

13. Atienda V, Prat Pastor JM, Peris Serra JL, Comín Clavijo M. Validación de un modelo de elementos finitos tridimensional del raquis lumbar mediante ensayos in vitro. Rev Esp Cir Ortop Traumatol. 2007;43(90):9041-8.

14. Gómez-Benito MJ, García-Aznar JM, Doblaré M. Finite element prediction of 
proximal femoral fracture patterns under different loads. J Biomech Eng. 2005;127(1):9-14.

15. Dragomir-Daescu D, Salas C, Uthamaraj S, Rossman T. Quantitative computed tomography-based finite element analysis predictions of femoral strength and stiffness depend on computed tomography settings. J Biomech. 2015;48(1):15361.

16. Rezaei A, Giambini H, Rossman T, Carlson KD, Yaszemski MJ, Lu L, et al. Are DXA/aBMD and QCT/FEA Stiffness and Strength Estimates Sensitive to Sex and Age? Ann Biomed Eng. 2017;45(12):2847-56.

17. Khoo BCC, Brown K, Cann C, Zhu K, Henzell S, Low V, et al. Comparison of QCTderived and DXA-derived areal bone mineral density and T scores. Osteoporos Int. 2009;20(9):1539-45.

18. Khoo BCC, Brown K, Zhu K, Pollock M, Wilson KE, Price Rl, et al. Differences in structural geometrical outcomes at the neck of the proximal femur using twodimensional DXA-derived projection (APEX) and three-dimensional QCT-derived (BIT QCT) techniques. Osteoporos Int. 2012;23(4):1393-8.

19. Dall'Ara E, Eastell R, Viceconti M, Pahr D, Yang L. Experimental validation of DXA-based finite element models for prediction of femoral strength. J Mech Behav Biomed Mater. 2016;63:17-25.

20. Bonnick S. HSA: Beyond BMD with DXA. Bone. 2007;41(1):S9-12.

21. Giner E, Arango C, Vercher A, Javier Fuenmayor F. Numerical modeling of the mechanical behaviour of an osteon with microcracks. J Mech Behav Biomed Mater. 2014;(37):109-24.

22. Morgan EF, Keaveny TM. Dependence of yield strain of human trabecular bone on anatomic site. J Biomech. 2001;34(5):569-77.

23. Marco M, Giner E, Larraínzar-Garijo R, Caeiro-Rey JR, Miguelez H. Análisis de la variación del comportamiento mecánico de la extremidad proximal del fémur mediante el método XFEM (eXtended Finite Element Method). Rev Osteoporos y Metab Miner. 2016;8(2):61-9. 
24. Kurz S, Pieroh P, Lenk M, Josten C, Böhme J. Three-dimensional reduction and finite element analysis improves the treatment of pelvic malunion reconstructive surgery. Medicine (Baltimore). 2017;96(42):e8136.

25. Vercher A, Giner E, Arango C, Tarancon J, Fuenmayor FJ. Homogenized stiffness matrices for mineralized collagen fibrils and lamellar bone using unit cell finite element models. Biomech Model Mechanobiol. 2014;13(2):437-49.

26. Lenich A, Bachmeier S, Dendorfer S, Mayr E, Nerlich M, Füchtmeier B. Development of a test system to analyze different hip fracture osteosyntheses under simulated walking. Biomed Tech Eng. 2012;57(2):113-9.

27. Lenich A, Bachmeier S, Prantl L, Nerlich M, Hammer J, Mayr E, et al. Is the rotation of the femural head a potential initiation for cutting out? A theoretical and experimental approach. BMC Musculoskelet Disord. 2011;12(1):79-86.

28. Herrera A, Panisello JJ, Ibarz E, Cegoñino J, Puértolas JA, Gracia L. Estudio densitométrico y con elementos finitos de la remodelación ósea tras la implantación de un vástago femoral anatómico no cementado. Rev Esp Cir Ortop Traumatol. 2008;52(5):269-82.

29. Palanca D, Peña E, Calvo B, Martínez M, Doblaré M. Análisis mediante elementos finitos del efecto de las roturas meniscales y meniscectomías. Rev Esp Cir Ortop Traumatol. 2007;48(4):298-303.

30. Seral García B, Cegoñino Banzo J, García Aznar JM, Doblaré Castellano M, Seral Iñigo F. Simulación en 3D con elementos finitos de un modelo de prótesis de rodilla. Rev Esp Cir Ortop Traumatol. 2003;47(1):64-72.

31. Larrainzar-Garijo R, García-Aznar J, Díez-Nicolas E. Análisis por simulación por elementos finitos del pie de la fórmula metatarsal de Maestro. Rev Pie y Tobillo. 2006;20(2):137-44.

Tabla 1

\begin{tabular}{|l|l|l|l|l|l|}
\hline Espécimen & Lado & Sexo & Edad & Altura $(\mathrm{cm})$ & Peso $(\mathrm{kg})$ \\
\hline
\end{tabular}




\begin{tabular}{|l|l|l|l|l|l|}
\hline 1 & Izquierdo & Mujer & 72 & 158 & 78 \\
\hline 2 & Derecho & Varón & 73 & 170 & 88 \\
\hline
\end{tabular}

Tabla 2

\begin{tabular}{|l|c|c|}
\hline Espécimen & Valor computacional & Valor experimental \\
\hline 1 & 25 & 12 \\
\hline 2 & 28 & 6 \\
\hline
\end{tabular}

Tabla 3

\begin{tabular}{|l|c|c|}
\hline Espécimen & Valor computacional & Valor experimental \\
\hline 1 & 6010 & 6572 \\
\hline 2 & 7120 & 8178 \\
\hline
\end{tabular}


Figura 1. Modelo 3D virtual por AEF de los especímenes estudiad

Click here to download high resolution image

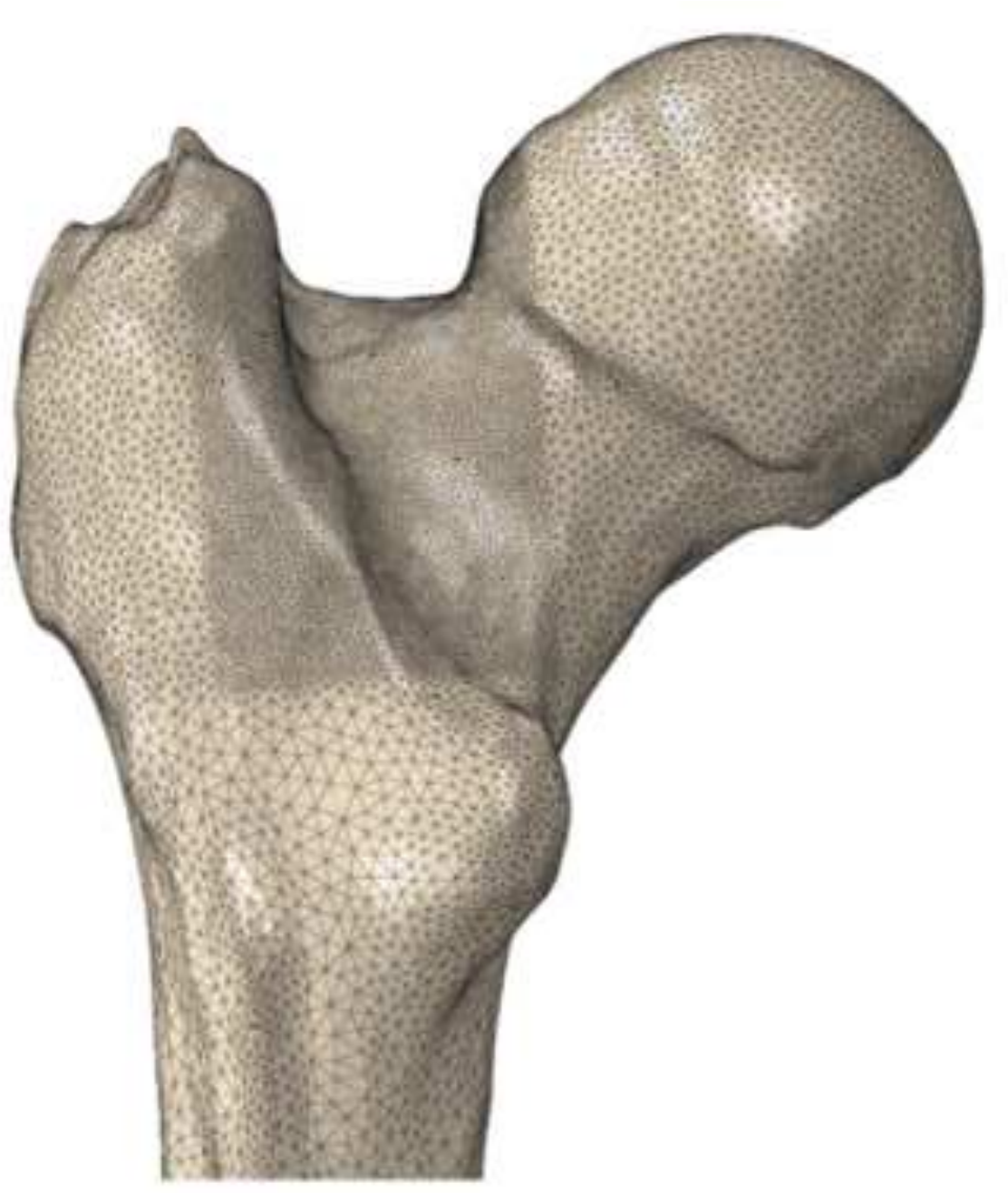

Espécimen 1

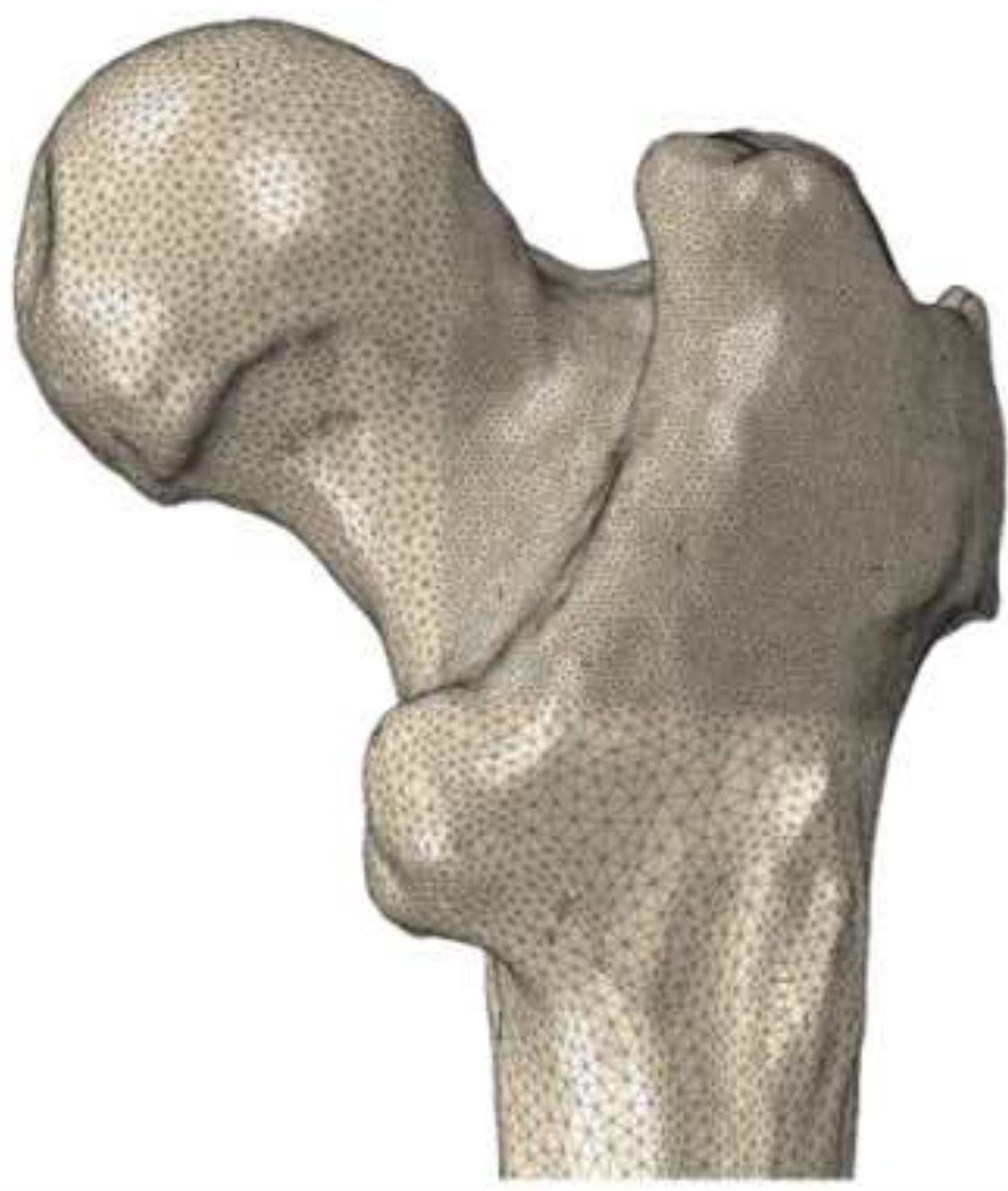

Espécimen 2 


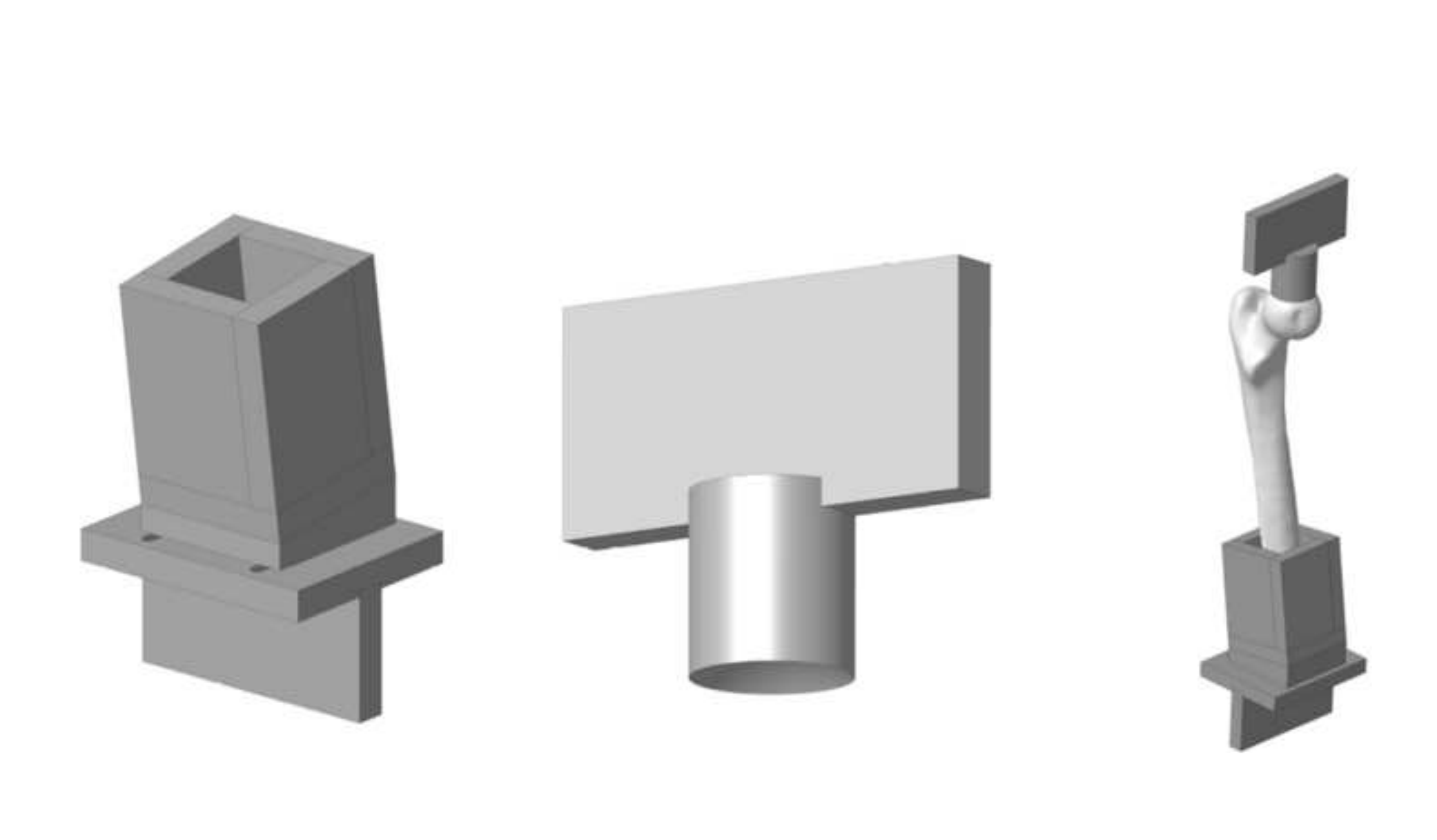



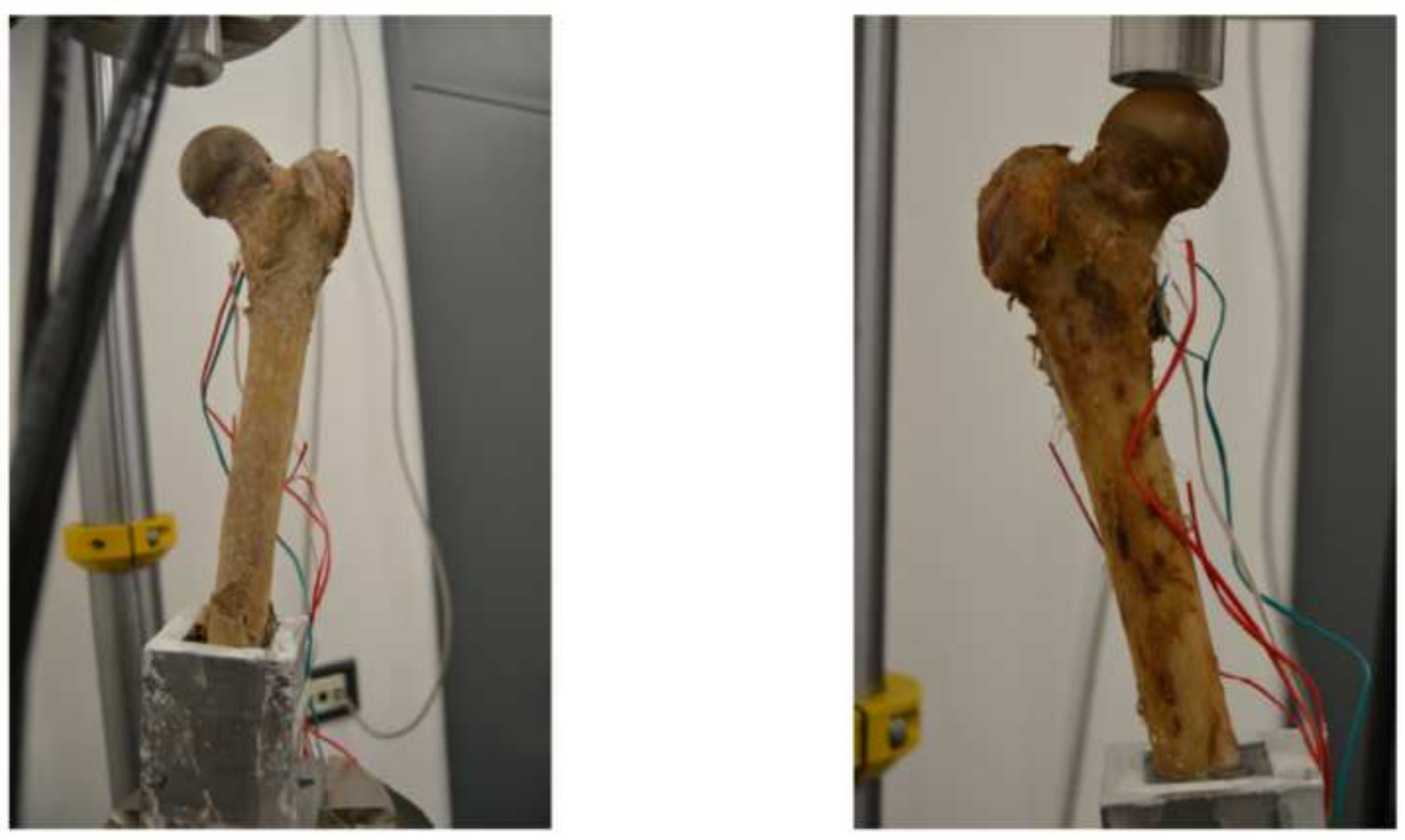 \\ Click here to download high resolution image
}

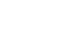


Click here to download high resolution image
Clion
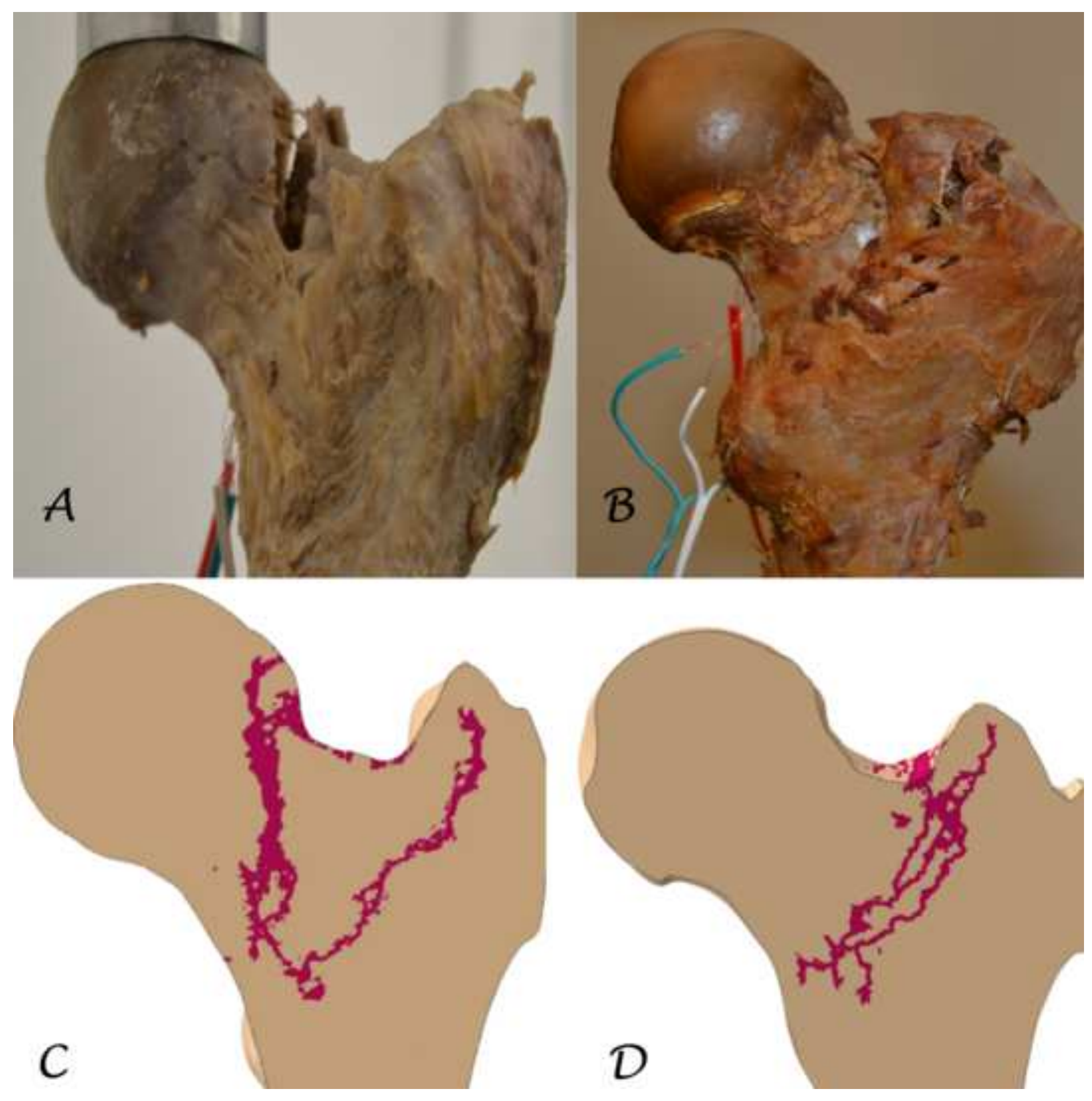

Figura 4. Se observa la propagación de la propagación de la frac

C 
Figura 5. muestra la comparación entre las deformaciones en ambo

Click here to download high resolution image

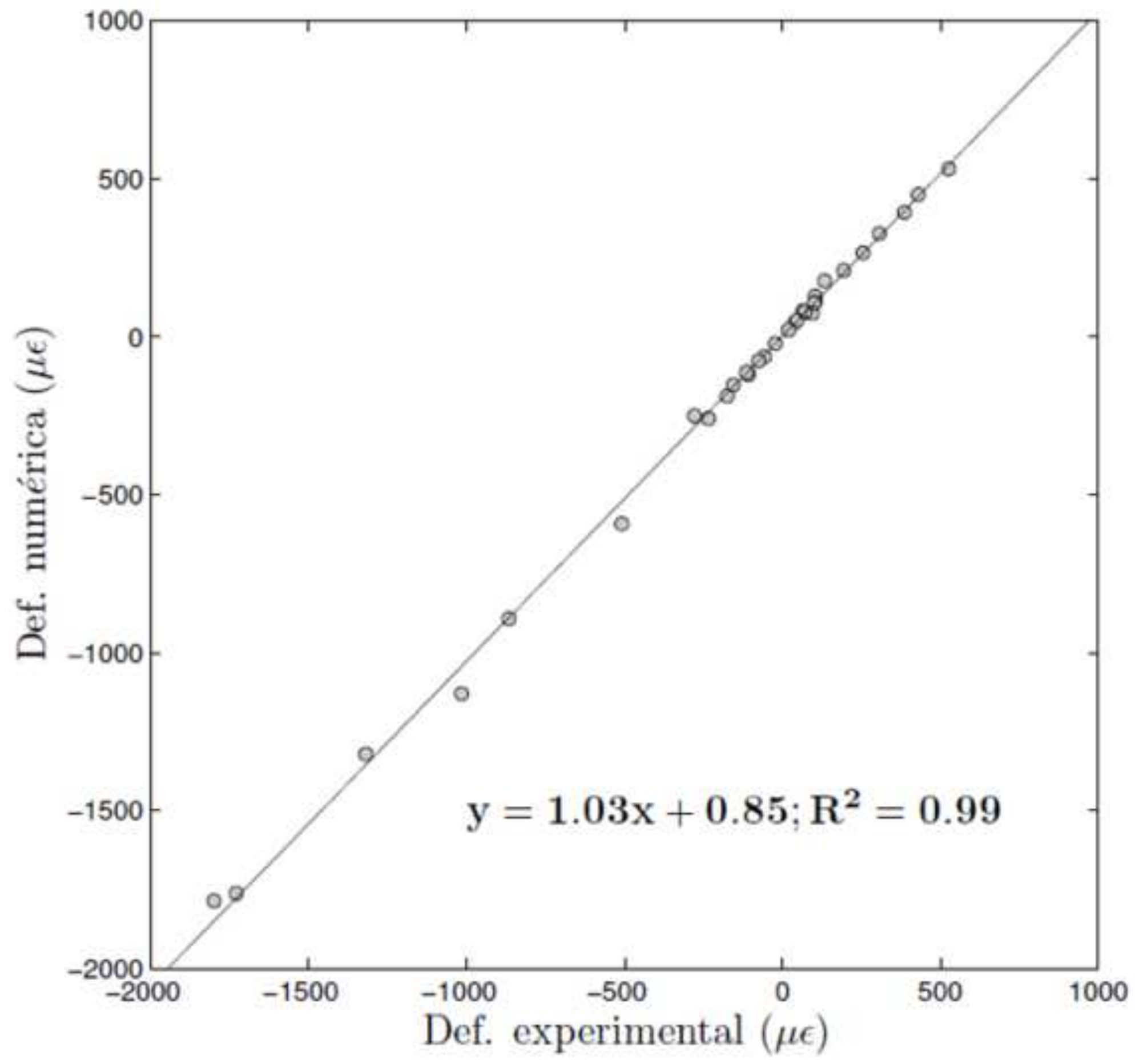

